\title{
Colon capsule endoscopy: European Society of Gastrointestinal Endoscopy (ESGE) Guideline
}

Authors

Institutions
C. Spada ${ }^{1}$, C. Hassan ${ }^{1}$, J. P. Galmiche ${ }^{2}$, H. Neuhaus ${ }^{3}$, J. M. Dumonceau ${ }^{4}$, S. Adler ${ }^{5}$, O. Epstein ${ }^{6}$, G. Gay ${ }^{7}$, M. Pennazio ${ }^{8}$, D. K. Rex ${ }^{9}$, R. Benamouzig ${ }^{10}$, R. de Franchis ${ }^{11}$, M. Delvaux ${ }^{7}$, J. Devière ${ }^{12}$, R. Eliakim ${ }^{13}$, C. Fraser ${ }^{14}$, F. Hagenmuller ${ }^{15}$, J. M. Herrerias ${ }^{16}$, M. Keuchel ${ }^{17}$, F. Macrae ${ }^{18}$, M. Munoz-Navas ${ }^{19}$, T. Ponchon ${ }^{20}$, E. Quintero ${ }^{21}$, M. E. Riccioni ${ }^{1}$, E. Rondonotti ${ }^{22}$, R. Marmo ${ }^{23}$, J. J. Sung ${ }^{24}$, H. Tajiri ${ }^{25}$, E. Toth ${ }^{26}$, K. Triantafyllou ${ }^{27}$, A. Van Gossum ${ }^{12}$, G. Costamagna

Institutions are listed at the end of article.
Bibliography

Dol http://dx.doi.org/

10.1055/s-0031-1291717

Published online: 2.3.2012

Endoscopy 2012; 44: 527-536

(c) Georg Thieme Verlag KG

Stuttgart · New York

ISSN 0013-726X

\section{Corresponding author}

\section{Spada, MD}

Digestive Endoscopy Unit

Università Cattolica del Sacro

Cuore

Largo A. Gemelli 8

00168 Roma

Italy

Fax: +39-06-30156581

cristianospada@gmail.com
PillCam colon capsule endoscopy (CCE) is an innovative noninvasive, and painless ingestible capsule technique that allows exploration of the colon without the need for sedation and gas insufflation. Although it is already available in European and other countries, the clinical indications for CCE as well as the reporting and work-up of de-

\section{Introduction \\ $\nabla$}

Colorectal cancer (CRC) represents a major cause of morbidity and mortality in Western countries [1]. Although CRC prevention, based on the identification and removal of precancerous adenomatous polyps as well as on downstaging of CRC that has already developed, has been shown to be highly effective in high quality randomized and observational studies [2-4], uptake in CRC screening programs is still disappointingly low, especially when compared with the high rate of participation in breast, cervical, and prostate screening programs $[5,6]$. Colon capsule endoscopy (CCE; Given Imaging Ltd, Yoqneam, Israel) is a new endoscopic technique which might have the potential for improving uptake of CRC screening [7-20]. It represents a minimally invasive and painless imaging system that allows exploration of the colon without the need for sedation and gas insufflation. Recently, a second-generation CCE device has been released that provides a higher number of images per second and a larger viewing angle [18-20].

Although CCE technology is now available on the market in many countries, its clinical indications as well as CCE reporting and post-CCE work-up have not yet been standardized. tected findings have not yet been standardized. The aim of this evidence-based and consensusbased guideline, commissioned by the European Society of Gastrointestinal Endoscopy (ESGE) is to furnish healthcare providers with a comprehensive framework for potential implementation of this technique in a clinical setting.

\section{Methods}

The European Society of Gastrointestinal Endoscopy (ESGE) commissioned this Guideline, which was then endorsed by its Governing Board. The guideline development process was similar to that used in creating other ESGE guidelines [21]: it included meetings, telephone conferences, and electronic media-based discussions among subgroups and members of the entire committee during October 2010 and March 2011.

Subgroups were formed, each being charged with a series of clearly defined key questions (see Appendix e1, available online). The committee chair (J.P.G.) worked with subgroup leaders (S.A., O.E., G.G., M.P.) to identify pertinent search terms that always included, as a minimum, "colon capsule endoscopy" as well as words pertinent to specific key questions. Searches were performed on Medline (via Pubmed), the Cochrane Library, Embase, and the internet. Articles were first selected by title; their relevance was then confirmed by review of the corresponding manuscript, and publications with content that was considered irrelevant were excluded. Additional articles, including abstracts presented at international conferences, were identified by manually searching the reference lists of retrieved papers. A central repository of selected literature was made available to all members of the guideline development group. Evidence tables were generated for each key question, summarizing the level of evidence of the available studies. The number of articles selected for each task force is indicated in the Evidence 
Table 1 Definitions of categories for evidence levels and recommendation grades used in this Guideline [21].

\begin{tabular}{|c|c|}
\hline \multicolumn{2}{|c|}{ Levels of evidence } \\
\hline $1++$ & $\begin{array}{l}\text { High quality meta-analyses, systematic reviews of RCTs, or RCTs } \\
\text { with a very low risk of bias }\end{array}$ \\
\hline $1+$ & $\begin{array}{l}\text { Well conducted meta-analyses, systematic reviews, or RCTs with } \\
\text { a low risk of bias }\end{array}$ \\
\hline $1-$ & Meta-analyses, systematic reviews, or RCTs with a high risk of bias \\
\hline $2++$ & $\begin{array}{l}\text { High quality systematic reviews of case - control or cohort } \\
\text { studies } \\
\text { High quality case - control or cohort studies with a very low risk of } \\
\text { confounding or bias and a high probability that the relationship is } \\
\text { causal }\end{array}$ \\
\hline $2+$ & $\begin{array}{l}\text { Well conducted case - control or cohort studies with a low risk } \\
\text { of confounding or bias and a moderate probability that the } \\
\text { relationship is causal }\end{array}$ \\
\hline $2-$ & $\begin{array}{l}\text { Case-control or cohort studies with a high risk of confounding or } \\
\text { bias and a significant risk that the relationship is not causal }\end{array}$ \\
\hline 3 & Nonanalytic studies, e.g. case reports, case series \\
\hline 4 & Expert opinion \\
\hline \multicolumn{2}{|c|}{ Grades of recommendation } \\
\hline A & $\begin{array}{l}\text { At least one meta-analysis, systematic review, or RCT rated as } \\
1++ \text {, and directly applicable to the target population; or } \\
\text { A body of evidence consisting principally of studies rated as } 1+, \\
\text { directly applicable to the target population, and demonstrating } \\
\text { overall consistency of results }\end{array}$ \\
\hline B & $\begin{array}{l}\text { A body of evidence including studies rated as } 2++ \text {, directly } \\
\text { applicable to the target population, and demonstrating overall } \\
\text { consistency of results; or } \\
\text { Extrapolated evidence from studies rated as } 1++ \text { or } 1+\end{array}$ \\
\hline $\mathrm{C}$ & $\begin{array}{l}\text { A body of evidence including studies rated as } 1-\text { or } 2+\text {, directly } \\
\text { applicable to the target population and demonstrating overall } \\
\text { consistency of results; or } \\
\text { Extrapolated evidence from studies rated as } 2++\end{array}$ \\
\hline $\mathrm{D}$ & $\begin{array}{l}\text { Evidence level } 2 \text { - or } 3 \text { or } 4 \text {; or } \\
\text { Extrapolated evidence from studies rated as } 2+\end{array}$ \\
\hline
\end{tabular}

$\mathrm{RCT}$, randomized controlled trial.

Note: The grade of recommendation relates to the strength of the evidence on which the recommendation is based. It does not reflect the clinical importance of the recommendation.

table (see Appendix e1 available online). For important outcomes, articles were individually assessed by using the Method for Evaluating Research and Guideline Evidence checklists as amended by the Scottish Intercollegiate Guidelines Network (SIGN) [22]. Evidence levels and recommendation grades used in this guideline were those recommended by the amended SIGN system ( Table 1) [22]. Subgroups agreed by online communication on draft proposals that were presented to the entire group for general discussion during a meeting held in February 2011 (Tarquinia, Italy). During the meeting and following discussion, competing proposals for wording of recommendations or assigning strength of evidence were resolved by formal voting. Consensus was defined as agreement on a statement by $\geq 66 \%$ of participants; this was then termed a consensus statement, and numbered for convenience in the document. Where the guideline development group was unable to achieve $\mathrm{a} \geq 66 \%$ agreement on a specific recommendation, the difference of opinion was formally recorded and the reasons for dissent were noted. The results of that discussion were incorporated into the subsequent guideline version and again discussed using email.

Searches were re-run in March 2011. Studies that were published after this date were not considered for inclusion. In March 2011, the final draft was sent to all individual ESGE members. After incorporation of comments made by the individual ESGE members, the manuscript was then sent to the Editorial Board of the journal Endoscopy for critique and international peer review. It underwent international peer review and the final version was approved by all members of the guideline development group.

Evidence statements and recommendations are stated in italics, key evidence statements and recommendations are in bold. This Guideline was issued in 2011 and will be considered for review in 2014 , or sooner if new evidence becomes available. Any updates to the guideline in the interim period will be noted on the ESGE website: http://www.esge.com/esge-guidelines.html.

\section{Summary of statements and recommendations}

$$
\nabla
$$

Indications and contraindications for colon capsule endoscopy (CCE)

CCE is feasible and safe and appears to be accurate when used in average-risk individuals (Evidence level $2++$, Recommendation grade (C). There is a lack of specific studies based in the setting of screening. CCE screening may be cost-effective if it increases screening uptake compared with colonoscopy (Evidence level 4, Recommendation grade $D$ ).

Patients at high risk for CRC, because of alarm symptoms or signs, or a family or personal history of CRC, are at increased risk of advanced colorectal neoplasia and cancer. These patients should be referred to colonoscopy. However, in patients for whom colonoscopy is inappropriate or not possible, the use of CCE could be discussed with the patient (Evidence level 4, Recommendation grade D). CCE is a feasible and safe tool for visualization of the colonic mucosa in patients with incomplete colonoscopy and without stenosis (Evidence level 3, Recommendation grade D). Randomized studies comparing CCE with radiological imaging or conventional endoscopic modalities are needed to confirm the efficacy of CCE in this setting and to better define the patients for whom CCE is most suitable (Evidence level 4, Recommendation grade $D$ ).

To date, there are insufficient data to support the use of CCE in the diagnostic work-up or in the surveillance of patients with suspected or known inflammatory bowel disease (IBD) (Evidence level 4, Recommendation grade $D$ ). On the basis of preliminary data, CCE may be useful to monitor inflammation in ulcerative colitis, which may help to guide therapy (Evidence level 4, Recommendation grade $D$ ).

Contraindications for CCE are similar to those for small-bowel capsule endoscopy. The use of sodium phosphate as a booster should be avoided in patients at increased risk of sodium phosphate toxicity. Other kinds of booster preparations are under investigation and may be considered in patients at increased risk of sodium phosphate toxicity (Evidence level 4, Recommendation grade D).

The risk of capsule retention with CCE is very low. In the case of capsule retention either in the small or the large bowel, endoscopy or surgery can be considered on the basis of the clinical background (EL, 3 Recommendation grade $D$ ).

\section{Bowel preparation for CCE}

Patients must follow a liquid diet the day before the procedure, whilst the role of low-residue diet is yet to be clarified (Evidence level 4, Recommendation grade $D$ ).

A total of $4 \mathrm{~L}$ of polyethylene glycol should be administered before the CCE procedure. A split-dosage regimen with intake the day before and on the day of the examination seems advisable to increase the tolerability and the efficacy of the preparation (Evidence level 4, Recommendation grade $D$ ). 
Table 2 Diagnostic accuracy of colon capsule endoscopy (CCE) for significant findings (polyps $6 \mathrm{~mm}$ in size, or 3 polyps).

\begin{tabular}{|c|c|c|c|c|c|}
\hline Author, year & $\begin{array}{l}\text { Patients with significant } \\
\text { findings, } \mathrm{n}(\%)\end{array}$ & Sensitivity & Specificity & PPV & NPV \\
\hline Eliakim, $2006[7]^{1}$ & $16(19)$ & $50 \%$ & $82 \%$ & $40 \%$ & $88 \%$ \\
\hline Schoofs, $2006[8]^{1}$ & $13(36)$ & $77 \%$ & $70 \%$ & $59 \%$ & $84 \%$ \\
\hline Van Gossum, $2009[9]^{1}$ & $87(27)$ & $64 \%$ & $84 \%$ & $60 \%$ & $86 \%$ \\
\hline Gay, $2010[11]^{1}$ & $67(53)$ & $76 \%$ & $76 \%$ & $78 \%$ & $74 \%$ \\
\hline Sacher-Huvelin, $2010[12]^{1}$ & $112(21)$ & $39 \%$ & $88 \%$ & $47 \%$ & $85 \%$ \\
\hline Pilz, $2010[13]^{1}$ & $6(10)$ & $50 \%$ & $75 \%$ & $19 \%$ & $93 \%$ \\
\hline Spada, $2011[14]^{1}$ & $13(33)$ & $62 \%$ & $85 \%$ & $67 \%$ & $82 \%$ \\
\hline Spada, $2011[15]^{1}$ & $7(15)$ & $100 \%$ & $95 \%$ & $78 \%$ & $100 \%$ \\
\hline Eliakim, $2009[18]^{2}$ & $18(19)$ & $89 \%$ & $76 \%$ & $46 \%$ & $97 \%$ \\
\hline Spada, $2011[20]^{2}$ & $45(41)$ & $84 \%$ & $64 \%$ & $62 \%$ & $85 \%$ \\
\hline All studies & $384(20)$ & $63 \%$ & $83 \%$ & $57 \%$ & $86 \%$ \\
\hline CCE-1 studies & $321(19)$ & $58 \%$ & $85 \%$ & $57 \%$ & $86 \%$ \\
\hline CCE-2 studies & $63(30)$ & $86 \%$ & $71 \%$ & $56 \%$ & $92 \%$ \\
\hline
\end{tabular}

PPV, positive predictive value; NPV; negative predictive value.

${ }^{1}$ First-generation colon capsule endoscopy (CCE-1) studies.

2 Second-generation (CCE-2) studies.

Booster preparations need to be given to improve capsule egestion rates and to complete visualization of the colonic mucosa (Evidence level 4, Recommendation grade $D$ ). In patients without contraindications to sodium phosphate, boosters based on sodium phosphate should be used (Evidence level 1+, Recommendation grade B). Lowdose sodium phosphate boosts have been shown to achieve an adequate CCE egestion rate and should be preferred over higher sodium phosphate doses (Evidence level 4, Recommendation grade D).

During the CCE procedure, the use of prokinetics is recommended when the capsule stays in the stomach longer than 1 hour (Evidence level 4, Recommendation grade D).

Written and oral information about CCE bowel preparation should be delivered by healthcare professionals (physician or nurse) involved in gastroenterology and with experience regarding the technique (Evidence level 4, Recommendation grade D).

\section{Reporting and work-up of CCE results}

The CCE report should provide information on the quality of preparation, technical details of the examination, completeness of the procedure, and on the significant findings (polyps/masses $\geq 6 \mathrm{~mm}$ or $\geq 3$ polyps, irrespective of size) in a standardized fashion (Evidence level 4, Recommendation grade D). In particular, size, morphology, and location should be separately described for each polyp. Extracolonic findings should also be reported when clinically meaningful (Evidence level 4, Recommendation grade D).

Patients found to have a polyp $\geq 6 \mathrm{~mm}$ at CCE, as well as those with $\geq 3$ polyps irrespective of size, should be sent for post-CCE colonoscopy for polypectomy (Evidence level 4, Recommendation grade D).

Patients without significant findings at CCE should repeat CCE or a different screening test after 5 years, unless bowel preparation at CCE was inadequate (Evidence level 4, Recommendation grade D). If overall bowel preparation was inadequate or CCE was incomplete, repetition of CCE or another colon imaging study should be considered (Evidence level 4, Recommendation grade D).

\section{Indications and contraindications for colon capsule endoscopy (CCE) \\ $\nabla$}

CCE is feasible and safe and appears to be accurate when used in average-risk individuals (Evidence level $2++$, Recommendation grade (). There is a lack of specific studies based in the setting of screening. CCE screening may be cost-effective if it increases screening uptake compared with colonoscopy (Evidence level 4, Recommendation grade $D$ ).

In the validation of noninvasive colorectal imaging tests for CRC screening and diagnosis, detection of advanced neoplasia has been regarded as a reliable intermediate end point. For instance, computed tomography (CT) colonography and immunochemical fecal tests have been extensively validated using this end point $[23,24]$. The validation process generally consists in assessing the accuracy of noninvasive tests for advanced neoplasia, using colonoscopy as an independent "gold standard" [25]. Because noninvasive colorectal imaging tests cannot provide a histological diagnosis, morphological criteria (i.e. polyp/mass $\geq 6 \mathrm{~mm}$ in size, or $\geq 3$ polyps) are accepted as surrogate markers of advanced neoplasia (high grade dysplasia, presence of villous component, or malignancy) $[7,8,23]$. Matching algorithms, based on size matching between the noninvasive test and the reference standard, have usually been incorporated in these studies to assure a reliable assessment of the new tests [7,8,23].

Of note, the accuracy values shown in these studies are independent from the prevalence of the disease. This means that values assessed in a population enriched with regard to advanced neoplasia remain accurate in settings where different prevalences may be expected, such as in asymptomatic average-risk populations.

The average sensitivity of the first generation of CCE (CCE-1) devices for significant findings ( $\geq 6 \mathrm{~mm}$ size, or $\geq 3$ polyps irrespective of size) was $58 \%$ [7 -17], substantially improving to $86 \%$ with the second generation CCE (CCE-2) devices ( Table 2) [18-20]. These rates are higher than the $50 \%$ cutoff for sensitivity that has been adopted by the American Cancer Society to define a test acceptable for screening purposes [25], and are comparable or superior to those of other noninvasive screening tests [26]. It should be emphasized that the ultimate aim of CRC screening is not to detect all the prevalent CRC (i.e. $100 \%$ sensitivity), but to 
reduce the prevalence significantly, also taking into account other variables, such as uptake, safety, costs, and complications. The apparently low specificity shown by CCE (see $\bullet$ Table 2; including CCE-2) has been shown to be mainly related to size mismatching between CCE and colonoscopy, resulting in an overestimation of size by CCE for diminutive lesions [18-20]. Although diminutive polyps do not represent a primary target for noninvasive imaging tests (see the work-up section below), it cannot be excluded at this stage that their detection may nevertheless be associated with CRC prevention, so that this low specificity should not be considered to be a significant obstacle to CCE implementation in a screening setting [20].

CCE has consistently been shown to be a very safe procedure: no major complication has been reported in over 1500 procedures, of which around $40 \%$ were in asymptomatic individuals [7-20]. CCE also appears to be a feasible procedure, with a very low rate of technical failures (i.e. $3 \%$ ) and a high capsule excretion rate of about $90 \%$ [7-20].

On the other hand, no data are available on the possible uptake of CCE in a screening context. A previous cost-effectiveness analysis has compared first-generation CCE with colonoscopy in a screening setting [27]. Although CCE was not a cost-effective alternative when equal uptake was assumed, it became an efficient option when it was assumed that uptake of CCE would be higher than that of colonoscopy for CRC screening, a premise that has not been demonstrated yet.

Patients with non-alarm symptoms do not appear to be at increased risk of colorectal neoplasia [28]. For this reason, noninvasive tests may be proposed in this setting as an alternative to colonoscopy [29]. Among noninvasive tests, however, imaging tests might be preferred over non-imaging ones (i.e. fecal tests), because of the ability, that may be regarded as clinically useful, to detect non-neoplastic conditions (e.g. vascular malformations). Among noninvasive imaging tests, CCE may be applicable in the context of screening because of the abovementioned considerations of feasibility, safety and accuracy. Although CCE has been shown to detect diverticular disease as well as inflammatory changes of the bowel mucosa [7-20], no study has specifically addressed its performance in detecting non-neoplastic alterations.

Patients at high risk for CRC, because of alarm symptoms or signs, or a family or personal history of CRC, are at increased risk of advanced colorectal neoplasia and cancer. These patients should be referred for colonoscopy. However, in patients for whom colonoscopy is inappropriate or not possible, the use of CCE could be discussed with the patient (Evidence level 4, Recommendation grade D). Patients with alarm symptoms (rectal bleeding, anemia, weight loss, intestinal subocclusion) are at increased risk of colorectal neoplasia. In particular, these patients are at a 5-10-fold increased risk of malignancy [30]. Patients with a positive fecal blood test are also at increased risk for CRC and advanced neoplasia [24-26]. In this setting, a test with a very high sensitivity is desirable and, therefore, colonoscopy or, alternatively, computed tomography (CT)-colonography or barium enema should be considered as the primary options [31]. CCE sensitivity for cancer is still unclear. As shown in $\bullet$ Table 3, the sensitivity of first-generation CCE (CCE-1) for CRC detection was suboptimal compared with that of colonoscopy [7-17]. Although CCE-2 would seem to be more sensitive (i.e. 4 cancers detected out of 4 true-positive cases, thus sensitivity $100 \%$ ) $[18,20]$ than CCE-1 for malignancy detection, data from a larger number of patients are needed before recommending the use of CCE-2 in this setting. Moreover,
Table 3 True-positive and false-negative diagnoses of histologically verified colorectal cancer reported with colon capsule endoscopy (CCE).

\begin{tabular}{|c|c|c|c|}
\hline Author, year & $\begin{array}{l}\text { Patients with } \\
\text { cancer, } \mathbf{n}\end{array}$ & $\begin{array}{l}\text { True- } \\
\text { positive }\end{array}$ & $\begin{array}{l}\text { False- } \\
\text { negative }\end{array}$ \\
\hline \multicolumn{4}{|c|}{ First-generation CCE studies } \\
\hline Eliakim, 2006 [7] & 0 & 0 & 0 \\
\hline Schoofs, 2006 [8] & 0 & 0 & 0 \\
\hline Van Gossum, 2009 [9] & 19 & 14 & 5 \\
\hline Sieg, 2009 [10] & 1 & 1 & 0 \\
\hline Gay, $2010[11]$ & 0 & 0 & 0 \\
\hline Sacher-Huvelin, 2010 [12] & 5 & 3 & 2 \\
\hline Pilz, 2010 [13] & 0 & 0 & 0 \\
\hline Spada, $2011[14]$ & 1 & 1 & 0 \\
\hline Spada, 2011 [15] & 1 & 1 & 0 \\
\hline \multicolumn{4}{|c|}{ Second-generation CCE studies } \\
\hline Eliakim, 2009 [18] & 1 & 1 & 0 \\
\hline Spada, 2011 [20] & 3 & 3 & 0 \\
\hline
\end{tabular}

subocclusive symptoms represent a contraindication to CCE because of the high risk of capsule retention.

CCE is a feasible and safe tool for visualization of the colonic mucosa in patients with incomplete colonoscopy and without stenosis (Evidence level 3, Recommendation grade D). Randomized studies comparing CCE with radiological imaging or conventional endoscopic modalities are needed to confirm the efficacy of CCE in this setting and to better define the patients for whom CCE is most suitable (Evidence level 4, Recommendation grade D).

The cecal intubation rate in colonoscopy has been reported to range widely from $60 \%$ to over $90 \%[32,33]$. Because of the risk of missed neoplasia in the nonvisualized colon, further tests may be advisable depending on patients' risk factors (i.e. left-sided polyps, family history, clinical indication). Such tests usually consist of radiological imaging (CT-colonography or barium enema) and/or colonoscopy using different endoscopes (pediatric or variable-stiffness colonoscopes, balloon-assisted enteroscopes) or with anaesthetist assistance [34]. The most frequent causes of incomplete colonoscopy include left-sided angulations caused by diverticular disease or post-surgical adhesions, extensive looping, or stenosing colorectal cancer [35]. In preliminary studies, CCE-1 has been shown to be feasible in this setting, although results for complete visualization of the colorectal mucosa have been conflicting, varying between $50 \%$ and $89 \%$ in small numbers of patients [36-38].

To date, there are insufficient data to support the use of CCE in the diagnostic work-up or in the surveillance of patients with suspected or known inflammatory bowel disease (IBD) (Evidence level 4, Recommendation grade D). On the basis of preliminary data, CCE may be useful to monitor inflammation in ulcerative colitis, which may help to guide therapy (Evidence level 4, Recommendation grade $D)$.

Inflammatory bowel diseases (IBDs) frequently involve the colorectal mucosa, with lesions reported in this location in $70-80 \%$ of patients with Crohn's disease and virtually always in those with ulcerative colitis [39]. Endoscopy is a suitable test for IBD diagnosis, because of its high sensitivity for the detection of mucosal lesions and its ability to sample the digestive mucosa. However, biopsy is usually required to improve test specificity, because of the possibility of false-positive results [40]. For this reason, colonoscopy is primarily recommended in this setting [41]. It is also recognized that treatment of ulcerative colitis should be 
tailored to the severity of colonic inflammation and that longlasting ulcerative colitis is associated with an increased risk of colorectal dysplasia and cancer [42,43]. Small-bowel capsule endoscopy provides a very high diagnostic yield for small-bowel mucosal lesions and its use is recommended in specific scenarios of IBD [40]. Similarly, CCE could be used to identify mucosal changes in the colorectal mucosa.

To date, the role of CCE in IBD has been evaluated in only one series [44]. Specifically, CCE has been compared with colonoscopy with the aim of evaluating its accuracy in monitoring colonic inflammation in patients with suspected or known ulcerative colitis. In this preliminary experience, CCE yielded encouraging results for detecting active ulcerative colitis (i.e., sensitivity $77 \%$, specificity $78 \%$ ) and substantial agreement with colonoscopy [44].

Contraindications for CCE are similar to those for small-bowel capsule endoscopy. The use of sodium phosphate as a booster should be avoided in patients at increased risk of sodium phosphate toxicity. Other kinds of booster preparations are under investigation and may be considered in patients at increased risk of sodium phosphate toxicity (Evidence level 4, Recommendation grade $D$ ).

CCE entails a similar technology to small-bowel capsule endoscopy, so that contraindications to small-bowel capsule endoscopy apply to CCE. The most frequent contraindications to capsule endoscopy are: dysphagia or swallowing disorder, prior major abdominal surgery of the gastrointestinal tract, known or suspected bowel obstruction, presence of a cardiac pacemaker or other implanted electromedical devices, and pregnancy [45]. In contrast to small-bowel capsule endoscopy, no data on safety of CCE in pediatric patients are available.

In addition to contraindications inherent to the capsule device, further contraindications can exist because the CCE procedure may imply the administration of sodium phosphate, although at lower doses than those usually used for bowel preparation for colonoscopy $[18,20]$. However, to be prudent, all the contraindications to use of sodium phosphate should be extended to CCE. In particular, sodium phosphate use should be avoided in elderly persons as well as in patients with hypovolemia, baseline kidney disease, bowel obstruction, or active colitis, and also in those who are using medications that affect renal perfusion or function [46]. In patients with contraindications to sodium phosphate, alternative booster agents (see section on bowel preparation) may be considered.

The risk of capsule retention with CCE is very low. In the case of capsule retention either in the small or the large bowel, endoscopy or surgery can be considered on the basis of the clinical background (Evidence level 3, Recommendation grade D).

Capsule retention is a major complication of small-bowel capsule endoscopy, with an overall incidence of $1-2 \%$, although reported rates widely vary between $0 \%$ and $21 \%$ depending on the indication for the examination [45]. Available studies with CCE, despite the larger capsule size, have not reported any capsule retention or sticking either in the small bowel or in the colon [7-20]. Of note, a case of CCE where a stuck colonic capsule was passed through a malignant colonic stricture and removed with the aid of flexible colonoscopy has recently been reported [47]. Removal of colonic capsule retained in the small bowel may require surgery, although removal by device-assisted enteroscopy may be an option [45]. Patients' symptoms and the possible pathology identified by CCE must also be taken into account in choosing the most appropriate treatment for capsule retention. Albeit infrequently, mild nausea, abdominal pain, and vomiting may occur during CCE and are attributed to colon preparation [7-18,20]. Antiemetic drugs have been empirically used to manage these situations.

\section{Bowel preparation for CCE}

\section{$\checkmark$}

Patients must follow a liquid diet the day before the procedure, whilst the role of low-residue diet is yet to be clarified (Evidence level 4, Recommendation grade $D$ ).

For conventional colonoscopy, a low-residue diet is often recommended during the 3 to 5 days before the bowel cleansing protocol itself. The aim is to decrease the amount of solid stool in the colon and thus reinforce the lavage effect of polyethylene glycol (PEG) solutions. This recommendation is reinforced when lowvolume cleansing protocols are proposed. However, very few studies have adequately addressed the efficacy of this low-residue diet before colonoscopy. On the other hand, when CCE is considered, recent experience seems to suggest that a clear liquid diet on the day before the procedure might improve the quality of bowel cleansing and consequently the diagnostic yield of CCE $[9,11,12,18,20]$. Therefore, diet recommendations (i.e., liquid diet the day before, low residue diet 3-5 days before CCE) have generally been adopted in the studies published so far. However, the prescription of diet restrictions over several days before the CCE may impair patient compliance with the cleansing protocol.

$A$ total of $4 L$ of PEG should be administered before the CCE procedure. A split-dosage regimen with intake the day before and on the day of the examination seems advisable to increase the tolerability and the efficacy of the preparation (Evidence level 4, Recommendation grade $D$ ).

Booster preparations need to be given to improve capsule egestion rates and to complete visualization of the colonic mucosa (Evidence level 4, Recommendation grade $D$ ). In patients without contraindications to sodium phosphate, boosters based on sodium phosphate should be used (Evidence level 1+, Recommendation grade B). Lowdose sodium phosphate boosts have been shown to achieve an adequate CCE egestion rate and should be preferred over higher sodium phosphate doses (Evidence level 4, Recommendation grade D).

During CCE, as the capsule is not equipped to insufflate the colon, aspirate liquids, wash the mucosal surface, and move actively along the gut, the cleansing protocol cannot be restricted to the time before the procedure but must be continued intraprocedurally $[7-15,18,20]$. The cleansing protocol for CCE aims at: (i) adequately cleansing the colonic mucosa, (ii) filling the colonic lumen with clear liquids to improve mucosal visualization and to decrease the number of air bubbles, and (iii) facilitating capsule progression so that it reaches the anal verge before the end of the battery life. Unpublished preliminary trials showed that a standard colonoscopy preparation could not achieve these tasks. Three trials demonstrated the efficacy of a protocol combining PEG and boosts with sodium phosphate to provide adequate bowel cleansing and high rates of complete colon examination $[9,10,12]$. Such a combined protocol has been applied in most subsequent studies although modifications in the timing and doses of the components have been reported. Starting from the experience with bowel cleansing for colonoscopy, lavage solutions with large volumes (3-4L) of PEG solutions have been used in most studies with CCE $[7-15,18,20]$. Very little experience with lower doses of PEG or substitutes such as sodium phosphate or magnesium citrate is currently available [48]. Studies demonstrating the equivalence of lower-volume cleansing and PEG pro- 


\begin{tabular}{|c|c|c|}
\hline & Rating & Description \\
\hline \multirow{8}{*}{$\begin{array}{l}\text { Cleansing level } \\
\text { scale }\end{array}$} & Poor & Inadequate \\
\hline & & Large amount of fecal residue precludes a complete examination \\
\hline & Fair & Inadequate but examination completed \\
\hline & & Enough feces or turbid fluid to prevent a reliable examination \\
\hline & Good & Adequate \\
\hline & & Small amount of feces or turbid fluid not interfering with examination \\
\hline & Excellent & Adequate \\
\hline & & No more than small bits of adherent feces \\
\hline \multirow{4}{*}{$\begin{array}{l}\text { Bubbles effect } \\
\text { scale }\end{array}$} & Significant & Bubbles that interfere with the examination \\
\hline & & More than $10 \%$ of surface area obscured by bubbles \\
\hline & Insignificant & No bubbles or bubbles that do not interfere with the examination \\
\hline & & Less than $10 \%$ of surface area obscured by bubbles \\
\hline
\end{tabular}

Table 4 Cleansing level scale and bubbles effect scale [51]. tocols for conventional colonoscopies cannot therefore be extended to CCE [49]. Two main split regimens of PEG have been proposed: " $3+1$ " (i.e., $3 \mathrm{~L}$ of PEG the day before and $1 \mathrm{~L}$ on the day of CCE) $[7-9,11,14]$, or "2+2" (i.e., 2 L of PEG the day before and $2 \mathrm{~L}$ on the day of CCE) $[15,18,20]$. Although no comparative study is available, the $2+2$ regimen seems to be more acceptable to patients and equally effective in terms of colon cleanliness.

To meet the specific goals of bowel cleansing for CCE discussed above, sodium phosphate boosts have been added to the usual PEG and diet recommendations used for conventional colonoscopy. The role of the boosts administered during capsule progression is not limited to increasing and/or maintaining colonic cleanliness but also includes a propulsive effect by means of a volume effect allowing the capsule to move in a watery environment. The propulsive effect of the sodium phosphate boosts results in an effective transit of the CCE device through the small and large bowels with a higher rate of capsule expulsion within the limited operating time of the capsule battery $[8-15,18,20]$. Magnesium sulfate has not been tested in this indication. However, a recent randomized study compared boosting with sodium phosphate to boosting with small volumes of PEG and concluded that the rate of capsule expulsion was higher with sodium phosphate [14]. The cumulative dose of sodium phosphate booster adopted initially in CCE studies was $75 \mathrm{~mL}[8,9]$. In order to reduce the risk of side effects from sodium phosphate administration, a lower dose ( 45 or $55 \mathrm{~mL}$, in total) has been used in the most recent studies on CCE-2, with no apparent decrease in capsule egestion rate $[18,20]$.

Potential adverse events caused by bowel preparation for CCE mainly appear to be related to sodium phosphate administration. Although no serious sodium phosphate-related adverse event has been reported up to now, after more than 1000 CCE examinations [7-18,20], use of sodium phosphate should be avoided in patients at higher risk of sodium phosphate toxicity (i.e. elderly persons, patients with hypovolemia, baseline kidney disease, bowel obstruction, or active colitis, patients on medications affecting renal perfusion or with function kidney failure, or having concomitant treatment with angiotensin-converting enzyme (ACE)-inhibitors, etc.) [46].

During the CCE procedure, the use of prokinetics is recommended when the capsule stays in the stomach longer than 1 hour (Evidence level 4, Recommendation grade D).

Prokinetics have been added to the protocol of bowel preparation for CCE mainly to stimulate the progression of the capsule in the upper gut, especially the stomach. In initial studies, tegaserod [7] and domperidone [8] were used. Domperidone was also used in multicenter trials $[9,11,12]$. No comparative study has so far been reported that assessed the usefulness of one dose of a pro- kinetic administered 15 minutes before capsule ingestion. With CCE-2, the data recorder allows real-time viewing of endoscopic images provided by the capsule; administration of prokinetics has thus been limited to cases where the capsule had not entered the small bowel within 1 hour after ingestion $[18,20]$.

Written and oral information about CCE bowel preparation should be delivered by healthcare professionals (physician or nurse) involved in gastroenterology and with experience regarding the technique (Evidence level 4, Recommendation grade $D$ ).

It is essential to strongly emphasize to the patient the need for good bowel preparation, since the quality of bowel preparation has been found to relate to the accuracy of the CCE procedure [9]. Experience with conventional colonoscopy shows that detailed information must be given to the patient before the procedure. The instructing person may be the endoscopist himself or herself, the referring physician, or an experienced and endoscopy-trained nurse [50]. Beyond the issues of quality and necessity of information with regard to bowel preparation, monitoring or "coaching" the patient during the cleansing protocol might be helpful to improve bowel cleansing.

\section{Reporting and work-up of CCE results $\nabla$}

The CCE report should provide information on the quality of preparation, technical details of the examination, completeness of the procedure, and on the significant findings (polyps/masses $\geq 6 \mathrm{~mm}$, or $\geq 3$ polyps irrespective of size) in a standardized fashion (Evidence level 4, Recommendation grade D). In particular, size, morphology, and location should be separately described for each polyp. Extracolonic findings should also be reported when clinically meaningful (Evidence level 4, Recommendation grade $D$ ).

Patients found to have a polyp $\geq 6 \mathrm{~mm}$ at CCE, as well as those with $\geq 3$ polyps irrespective of size, should be sent for post-CCE colonoscopy for polypectomy (Evidence level 4, Recommendation grade D).

Patients without significant findings at CCE should repeat CCE or a different screening test after 5 years, unless bowel preparation at CCE was inadequate (Evidence level 4, Recommendation grade D).

Quality of bowel preparation has been related to CCE accuracy for relevant lesions [9]. Mucosal visualization by CCE may be hampered by feces, turbid fluids, or bubbles. Scoring on all these items has recently been included in a grading system that allowed evaluation of colon cleansing for CCE with good interobserver agreement ( Table4) [51].

A CCE examination may be incomplete, mainly because a slow colonic transit that may mean that the battery is exhausted while the CCE device is still in the colon. Therefore, some colorectal 
segments (usually the left colon) may not be visualized by CCE, potentially leading to false-negative results.

Most colonic polyps discovered at screening are diminutive, with negligible risk of harboring advanced features (high grade dysplasia, villous component, or malignancy) [52-55]. Moreover, $40 \%$ of diminutive colonic polyps are hyperplastic rather than adenomatous [56]. Diminutive lesions identified by a noninvasive test may also be missed by the post-test colonoscopy, because of the imperfect sensitivity of the latter for diminutive lesions $[57,58]$. By extrapolating data from CT colonography studies that modelled the impact of colonoscopy or continued surveillance for diminutive polyps discovered at CT-colonography, it can be concluded that referral for removal of diminutive lesions found at CCE might carry an unjustified burden of costs and complications relative to a minimal gain in clinical efficacy $[59,60]$. Moreover, studies on second-generation CCE only provide accuracy data relating to lesions $\geq 6 \mathrm{~mm}$ in size, its specificity for diminutive lesions being largely unknown $[18,20]$. Thus, the only exception regarding post-CCE referral for diminutive polyps is the simultaneous presence of at least 3 of these lesions. Polyp multiplicity has appeared as a strong predictive factor of subsequent advanced neoplasia development in post-polypectomy follow-up studies [61]. Most advanced neoplasias have been shown to be restricted to the relatively small proportion of patients with polyps $\geq 6 \mathrm{~mm}$ in size [55]. Consequently, post-CCE colonoscopy referral of such patients may be expected to lead to a substantial reduction of the prevalence of advanced neoplasia in patients initially evaluated with CCE. Although it has been shown that patients with polyps of size $6-9 \mathrm{~mm}$ may be safely followed up for a relatively short period of time [62-64], there is no evidence that CCE repetition after 2-3 years may lead to re-identification of the previously unremoved polyp.

The efficacy of a noninvasive test such as CCE ultimately depends on the identification and removal/biopsy sampling at post-CCE colonoscopy of the lesions found at CCE. For this reason, it is critically important that the size, morphology, and location of lesions should be accurately described, to facilitate the post-CCE identification at colonoscopy. The Paris classification may be extended to CCE to standardize polyp reporting [65].

Similarly to small-bowel capsule endoscopy, CCE may detect clinically important extracolonic findings, such as preneoplastic (i.e. Barrett esophagus), ulcerative, or malignant processes of the upper gastrointestinal tract or the small bowel. Although the impact of CCE diagnosis on the course of these extracolonic diseases has not been addressed, appropriate patient care requires that the presence of clinically important abnormalities should be identified and effectively communicated.

The long-term efficacy of CCE with regard to CRC prevention is unknown at present. A 5-year interval before repeating a new screening test has been recommended for other noninvasive imaging procedures, such as flexible sigmoidoscopy, CT colonography, or barium enema [25]. Considering that the sensitivity of CCE appears to be comparable to that of these tests, the 5year interval may be extended to CCE by analogy.

If overall bowel preparation was deemed inadequate or CCE was incomplete, repetition of an imaging study should be considered [Evidence level 4, Recommendation grade $D$ ].

There is an association between the cleanliness level and the accuracy of CCE for detecting polyps [9]. False-negative CCE findings have also been related to incomplete visualization of the colorectal mucosa because of CCE battery exhaustion [16]. Although possible predictors of inadequate bowel preparation at
CCE have not yet been analyzed, the effectiveness of bowel preparation has been shown at colonoscopy to depend on a number of factors, the most important of which is compliance [66-68]. Other independent risk factors for incomplete colon preparation include a history of constipation, inpatient status, use of antidepressants, and male gender [68]. In the case of inadequate colon cleansing at CCE or of incomplete visualization of the colorectal mucosa, it may not be possible to exclude even large lesions in the poorly visualized or unvisualized areas. For this reason, based on clinical background, repetition of CCE or a different imaging test may be considered. For a follow-on procedure, whichever one is recommended, the potential reasons for inadequate CCE preparation should be addressed. Poor compliance might be addressed by better education and supervision, and by changing the type and/or intensity of bowel preparation.

\section{Use of this Guideline}

$\nabla$

The aim of this Guideline is to provide caregivers with a comprehensive framework on how to implement and practice CCE in a clinical setting. ESGE Guidelines represent a consensus of best practice based on the available evidence at the time of preparation. They may not apply in all situations and should be interpreted in the light of specific clinical situations and resource availability. Further controlled clinical studies may be needed to clarify aspects of these statements, and revision may be necessary as new data appear. Clinical consideration may justify a course of action at variance to these recommendations. ESGE Guidelines are intended to be an educational device to provide information that may assist endoscopists in providing care to patients. They are not rules and should not be construed as establishing a legal standard of care or as encouraging, advocating, requiring, or discouraging any particular treatment.

Competing interests: Given Imaging funded the consensus meeting in Tarquinia. C. Spada, C. Hassan, J.P. Galmiche, S. Adler, M. Pennazio, D. K. Rex, R. de Franchis, and E. Toth are consultants for Given Imaging. M. Keuchel, F. Macrae, F. Hagenmueller, T. Ponchon, J.J. Sung, and G. Costamagna have received research support and are consultants for Given Imaging. R. Benamouzig and J. Devière received research support from Given Imaging. H. Neuhaus, J. M. Dumonceau, O. Epstein, G. Gay, M. Delvaux, R. Eliakim, C. Fraser, J. M. Herrerias, M. Munoz-Navas, E. Quintero, M.E. Riccioni, E. Rondonotti, R. Marmo, H. Tajiri, K. Triantafyllou, and A. Van Gossum declare no conflict of interest to disclose.

\section{Institutions}

${ }^{1}$ Digestive Endoscopy Unit, Catholic University, Rome, Italy

2 Department of Gastroenterology and Hepatology, Nantes University, Nantes, France

${ }^{3}$ Department of Gastroenterology, Evangelisches Krankenhaus, Düsseldorf, Germany

${ }^{4}$ Service of Gastroenterology and Hepatology, Geneva University Hospitals, Geneva, Switzerland

${ }^{5}$ Department of Gastroenterology, Bikur Holim Hospital; Jerusalem, Israel

${ }^{6}$ Department of Gastroenterology, Royal Free and University College Medical School, London, UK

${ }^{7}$ Department of Hepato-Gastroenterology, CHU Strasbourg, Strasbourg, France

${ }^{8}$ Division of Gastroenterology 2, San Giovanni Battista University Teaching Hospital, Turin, Italy

${ }^{9}$ Department of Gastroenterology, Indiana University Hospital, Indianapolis, USA

${ }^{10}$ Department of Gastroenterology, Avicenne Hospital, University of Paris, Bobigny, France 
${ }^{11}$ Gastroenterology Unit, Luigi Sacco University Hospital, Milan, Italy

12 Department of Gastroenterology, Erasme Hospital, Université Libre de Bruxelles, Brussels, Belgium

${ }^{13}$ Chaim Sheba Medical Center, Tel-Aviv, Israel

${ }^{14}$ Department of Gastroenterology, St Mark's Hospital, London, UK

${ }^{15}$ Department of Medicine I, Altona General Hospital, Hamburg, Germany

${ }^{16}$ Gastroenterology Service, Virgen Macarena University Hospital, Seville, Spain

${ }^{17}$ Clinic for Internal Medicine, Bethesda Krankenhaus Bergedorf, Hamburg, Germany

${ }^{18}$ Department of Gastroenterology and Clinical Nutrition Service, Royal Melbourne Hospital, Melbourne, Australia

${ }^{19}$ Digestive Endoscopy Unit, Clinica Universitaria de Navarra, Pamplona, Spain

${ }^{20}$ Department of Gastroenterology, Hôpital Edouard Herriot, Lyon, France

${ }^{21}$ Department of Gastroenterology, Hospital Universitario de Canarias, Tenerife, Spain

${ }^{22}$ Gastroenterology Unit, Ospedale Valduce, Como, Italy

${ }^{23}$ Division of Gastroenterology, Curto Hospital, Polla, Italy

${ }^{24}$ Department of Medicine and Therapeutics, Division of Gastroenterology, Prince of Wales Hospital, Shatin, N.T., Hong Kong

${ }^{25}$ Division of Gastroenterology and Hepatology, Department of Internal Medicine, The Jikei University School of Medicine, Tokyo, Japan

${ }^{26}$ Endoscopy Unit, Skane University Hospital, Lund University, Malmö, Sweden

${ }^{27}$ Hepatogastroenterology Unit, 2nd Department of Internal MedicinePropaedeutic, Attikon University General Hospital, Athens University, Athens, Greece

\section{References}

1 Ferlay J, Autier P, Boniol $M$ et al. Estimates of the cancer incidence and mortality in Europe in 2006. Ann Oncol 2007; 18: 581 - 592

2 Atkin WS, Edwards R, Kralj-Hans I et al. Once-only flexible sigmoidoscopy screening in prevention of colorectal cancer: a multicentre randomised controlled trial. Lancet 2010; 375: 1624-1633

3 Winawer SJ, Zauber AG, Ho MN. The National Polyp Study Workgroup. et al. Prevention of colorectal cancer by colonoscopic polypectomy. $\mathrm{N}$ Engl J Med 1993; 329: 1977-1981

4 Citarda F, Tomaselli $G$, Capocaccia $R$ et al. Efficacy in standard clinical practice of colonoscopic polypectomy in reducing colorectal cancer incidence. Gut 2001; 48: 812-815

5 Segnan N, Senore C, Andreoni B et al. Comparing attendance and detection rate of colonoscopy with sigmoidoscopy and FIT for colorectal cancer screening. Gastroenterology 2007; 132: 2304-2312

6 Lisi D, Hassan C, Crespi $M$ et al. Participation to colorectal cancer screening with FOBT and colonoscopy: An Italian, multicentre, randomized population study. Dig Liver Dis 2010; 42: 371 - 376

7 Eliakim R, Fireman Z, Gralnek IM et al. Evaluation of the PillCam Colon capsule in the detection of colonic pathology: results of the first multicenter, prospective, comparative study. Endoscopy 2006; 38: 963 - 970

8 Schoofs N, Devière J, Van Gossum A et al. PillCam colon capsule endoscopy compared with colonoscopy for colorectal tumor diagnosis: a prospective pilot study. Endoscopy 2006; 38: 971 - 977

9 Van Gossum A, Navas MM, Fernandez-Urien I et al. Capsule endoscopy versus colonoscopy for the detection of polyps and cancer. $\mathrm{N}$ Engl J Med 2009; 361: 264-270

10 Sieg A, Friedrich $K$, Sieg $U$ et al. Is PillCam COLON capsule endoscopy ready for colorectal cancer screening? A prospective feasibility study in a community gastroenterology practice Am J Gastroenterol 2009; 104: $848-854$

11 Gay G, Delvaux $M$, Frederic $M$ et al. Could the colonic capsule PillCam Colon be clinically useful for selecting patients who deserve a complete colonoscopy?: results of clinical comparison with colonoscopy in the perspective of colorectal cancer screening Am J Gastroenterol 2010; 105: 1076 - 1086

12 Sacher-Huvelin S, Coron E, Gaudric M et al. Colon capsule endoscopy vs. colonoscopy in patients at average or increased risk of colorectal cancer. Aliment Pharmacol Ther 2010; 32: 1145 - 1153

13 Pilz JB, Portmann S, Peter S et al. Colon capsule endoscopy compared to conventional colonoscopy under routine screening conditions. BMC Gastroenterol 2010; 10: 66

14 Spada C, Riccioni ME, Hassan C et al. PillCam colon capsule endoscopy: a prospective, randomized trial comparing two regimens of preparation. J Clin Gastroenterol 2011; 45: 119-124

15 Spada $C$, Hassan $C$, Ingrosso $M$ et al. A new regimen of bowel preparation for PillCam colon capsule endoscopy: a pilot study. Dig Liver Dis 2011; 43: $300-304$
16 Spada C, Hassan C, Marmo R et al. Meta-analysis shows colon capsule endoscopy is effective in detecting colorectal polyps. Clin Gastroenterol Hepatol 2010; 8: 516-522

17 Rokkas T, Papaxoinis $K$, Triantafyllou Ket al. A meta-analysis evaluating the accuracy of colon capsule endoscopy in detecting colon polyps. Gastrointest Endosc 2010; 71: 792 - 798

18 Eliakim R, Yassin K, Niv Y et al. Prospective multicenter performance evaluation of the second-generation colon capsule compared with colonoscopy. Endoscopy 2009; 41: 1026-1031

19 Spada C, Hassan C, Riccioni ME et al. False positive at colon capsule endoscopy or false negative at conventional colonoscopy? Endoscopy 2010; 42: $427-428$

20 Spada C, Hassan C, Munoz-Navas M et al. Second-generation colon capsule compared with colonoscopy. Gastrointest Endosc 2011; 74: 581 589

21 Dumonceau JM, Andriulli A, Deviere J et al. European Society of Gastrointestinal Endoscopy (ESGE) Guideline: prophylaxis of post-ERCP pancreatitis. Endoscopy 2010; 42: $503-515$

22 Harbour R, Miller J. A new system for grading recommendations in evidence based guidelines. BMJ 2001; 323: 334-336

23 Johnson $C D$, Chen $M H$, Toledano AY et al. Accuracy of CT colonography for detection of large adenomas and cancers. N Engl J Med 2008; 359: $1207-1217$

24 Hundt S, Haug $U$, Brenner $H$. Comparative evaluation of immunochemical fecal occult blood tests for colorectal adenoma detection. Ann Intern Med 2009; 150: 162 - 169

25 Levin B, Lieberman DA, McFarland B et al. Screening and surveillance for the early detection of colorectal cancer and adenomatous polyps, 2008: a joint guideline from the American Cancer Society, the US Multi-Society Task Force on Colorectal Cancer, and the American College of Radiology. Gastroenterology 2008; 134: 1570-1595

26 Lieberman DA. Clinical practice. Screening for colorectal cancer. N Engl J Med 2009; 361: 1179-1187

27 Hassan C, Zullo A, Winn S et al. Cost-effectiveness of capsule endoscopy in screening for colorectal cancer. Endoscopy 2008; 40: 414-421

28 Rex DK, Mark D, Clarke B et al. Flexible sigmoidoscopy plus air-contrast barium enema versus colonoscopy for evaluation of symptomatic patients without evidence of bleeding. Gastrointest Endosc 1995; 42: $132-138$

29 Pickhardt PJ, Choi JR, Hwang I et al. Computed tomographic virtual colonoscopy to screen for colorectal neoplasia in asymptomatic adults. N Engl J Med 2003; 349: 2191 - 2200

30 Goulston KJ, Cook I, Dent OF. How important is rectal bleeding in the diagnosis of bowel cancer and polyps? Lancet 1986; 2: 261 - 265

31 American Society for Gastrointestinal Endoscopy. Appropriate use of gastrointestinal endoscopy. Gastrointest Endosc 2000; 52: 831 - 837

32 Bowles $C J$, Leicester $R$, Romaya $C$ et al. A prospective study of colonoscopy practice in the UK today: are we adequately prepared for national colorectal cancer screening tomorrow? Gut 2004; 53: 277-283

33 Lieberman DA, Weiss DG, Bond JH. Veterans Affairs Cooperative Study Group 380. et al. Use of colonoscopy to screen asymptomatic adults for colorectal cancer. N Engl J Med 2000; 343: 162 - 168

34 Rex DK. Achieving cecal intubation in the very difficult colon. Gastrointest Endosc 2008; 67: 938-944

35 Hanson ME, Pickhardt PJ, Kim DH et al. Anatomic factors predictive of incomplete colonoscopy based on findings at CT colonography. AJR Am J Roentgenol 2007; 189: 774-779

36 Spada C, Riccioni ME, Petruzziello $L$ et al. The new PillCam Colon capsule: difficult colonoscopy? No longer a problem? Gastrointest Endosc 2008; 68: $807-808$

37 Triantafyllou K, Tsibouris P, Kalantzis $C$ et al. PillCam Colon capsule endoscopy does not always complement incomplete colonoscopy. Gastrointest Endosc 2009; 69: 572-576

38 Triantafyllou $K$, Viazis $N$, Tsibouris $P$ et al. Colon capsule endoscopy complements incomplete colonoscopy in clinical practice. Endoscopy 2010; $42: 01 \mathrm{~A} 27$

39 Abraham C, Cho JH. Inflammatory bowel disease. N Engl J Med 2009; 361: $2066-2078$

40 Bourreille A, Ignjatovic A, Aabakken L et al. Role of small-bowel endoscopy in the management of patients with inflammatory bowel disease: an international OMED-ECCO consensus. Endoscopy 2009; 41: 618 637

41 Strange EF, Travis SPL, Vermeire S et al. European evidence-based Consensus on the diagnosis and management of ulcerative colitis: Definitions and diagnosis. J Crohn's Colitis 2008; 2: 1-23 
42 Lichtenstein GR, Rutgeerts $P$. Importance of mucosal healing in ulcerative colitis. Inflamm Bowel Dis 2010; 16: 338-346

43 D'Haens GR, Panaccione R, Higgins PD et al. The London Position Statement of the World Congress of Gastroenterology on Biological Therapy for IBD with the European Crohn's and Colitis Organization: when to start, when to stop, which drug to choose, and how to predict response? Am J Gastroenterol 2011; 106: 199-212

44 Sung JJY, Ching JY, Leung WK et al. Assessment of colonic inflammatory lesions and ulcerative colitis with PillCam Colon capsule endoscopy compared to colonoscopy. Endoscopy 2008; 40: A199

45 Ladas SD, Triantafyllou K, Spada C et al. European Society of Gastrointestinal Endoscopy (ESGE): recommendations (2009) on clinical use of video capsule endoscopy to investigate small-bowel, esophageal and colonic diseases. Endoscopy 2010; 42: 220-227

46 US Food and Drugs Administration. Oral sodium phosphate (OSP) products for bowel cleansing (marketed as Visicol and OsmoPrep, and oral sodium phosphate products available without a prescription). Available at: www.fda.gov/Drugs/DrugSafety/PostmarketDrugSafetyInformationforPatientsandProviders/ucm103354.htm (Accessed: 11 March 2011)

47 Rácz I, Jánoki M, Saleh H. Colon cancer detection by 'rendezvous colonoscopy': successful removal of stuck colon capsule by conventional colonoscopy. Case Rep Gastroenterol 2010; 4: 19-24

48 Kakugawa Y, Saito $Y$, Saito $S$ et al. Evaluation of newly modified bowel preparation method as a less invasive procedure in conducting colon capsule endoscopy. ICCD Proceedings, Paris, August 27-28 2010 [abstract]

49 Marmo R, Rotondano G, Riccio G et al. Effective bowel cleansing before colonoscopy: a randomized study of split-dosage versus non-split dosage regimens of high-volume versus low-volume polyethylene glycol solutions. Gastrointest Endosc 2010; 72: 313 - 320

50 Segarajasingam DS, Pawlik J, Forbes GM. Informed consent in direct access colonoscopy. J Gastroenterol Hepatol 2007; 22: 2081 - 2085

51 Leighton J, Rex D. A grading scale to evaluate colon cleansing for the PillCam COLON capsule: a reliability study. Endoscopy 2011; 43: $123-127$

52 Lieberman D, Moravec M, Holub J et al. Polyp size and advanced histology in patients undergoing colonoscopy screening: Implications for CT colonography. Gastroenterology 2008; 135: 1100-1105

53 Bond $\mathrm{JH}$. Clinical relevance of the small colorectal polyp. Endoscopy 2001; 33: 454-457

54 Church JM. Clinical significance of small colorectal polyps. Dis Colon Rectum 2004; 47: 481 - 485
55 Hassan C, Pickhardt PJ, Kim DH et al. Systematic review: Distribution of advanced neoplasia according to polyp size at screening colonoscopy. Aliment Pharmacol Ther 2010; 31: 210-217

56 Weston AP, Campbell DR. Diminutive colonic polyps: histopathology, spatial distribution, concomitant significant lesions, and treatment complications. Am J Gastroenterol 1995; 90: 24-28

57 van Rijn JC, Reitsma JB, Stoker J et al. Polyp miss rate determined by tandem colonoscopy: a systematic review. Am J Gastroenterol 2006; 10 : $343-350$

58 Rex DK, Cutler CS, Lemmel GT et al. Colonoscopic miss rates of adenomas determined by back to back colonoscopies. Gastroenterology 1997; $112: 24-28$

59 Pickhardt PJ, Hassan C, Laghi A et al. Cost-effectiveness of colorectal cancer screening with computed tomography colonography - the impact of not reporting diminutive lesions. Cancer 2007; 109: 2213 2221

60 Pickhardt PJ, Hassan C, Laghi $H$ et al. Small and diminutive polyps detected at screening CT colonography: a decision analysis for referral to colonoscopy. AJR Am J Roentgenol 2008; 190: 136-144

61 Martínez ME, Baron JA, Lieberman DA et al. A pooled analysis of advanced colorectal neoplasia diagnoses after colonoscopic polypectomy. Gastroenterology 2009; 136: 832-841

62 Hoff G, Foerster A, Vatn MH et al. Epidemiology of polyps in the rectum and colon. Recovery and evaluation of unresected polyps 2 years after detection. Scand J Gastroenterol 1986; 21: 853-862

63 Hofstad B, Vatn MH, Andersen SN et al. Growth of colorectal polyps: redetection and evaluation of unresected polyps for a period of three years. Gut 1996; 39: 449-456

64 Pickhardt PJ, Hassan C, Laghi A et al. Clinical management of small (6to 9-mm) polyps detected at screening CT colonography: a cost-effectiveness analysis. AJR Am J Roentgenol 2008; 191: 1509-1516

65 The Paris endoscopic classification of superficial neoplastic lesions: esophagus, stomach, and colon: November 30 to December 1, 2002. Gastrointest Endosc 2003; 58: S3-43

$66 \mathrm{Hsu} C W$, Imperiale TF. Meta-analysis and cost comparison of polyethylene glycol lavage versus sodium phosphate for colonoscopy preparation. Gastrointest Endosc 1998; 48: 276-282

67 Belsey J, Epstein O, Heresbach D. Systematic review: oral bowel preparation for colonoscopy. Aliment Pharmacol Ther 2007; 25: 373-384

68 Ness RM, Manam R, Hoen $H$ et al. Predictors of inadequate bowel preparation for colonoscopy. Am J Gastroenterol 2001; 96: 1797-1802

Appendix e1 and e2 are available online:

online content viewable at:

www.thieme-connect.de/ejournals/abstract/endoscopy/

doi/10.1055/s-0031-1291717 